\title{
Primary Benign Phyllodes Tumor of The Vulva: Case Report and Review of Literature
}

\author{
Asuman Kilitci ${ }^{1}$ (D), Okan Arioz ${ }^{2}$ (1) \\ ${ }^{1}$ Department of Pathology, Ahi Evran University School of Medicine, Kırşehir, Turkey \\ ${ }^{2}$ Department of Gynecology and Obstetrics, Ahi Evran University Training and Research Hospital, Kurşehir, Turkey
}

\begin{abstract}
Phyllodes tumor (PT) of the vulva is very rarely seen and has been reported in only 17 cases in English literature. It is still uncertain that proliferative mammary gland lesions including PT in the anogenital region originate from ectopic breast tissue or from local adnexal structures. We report a case of primary benign PT of the vulva in a 41-year-old female patient. Microscopic examination revealed biphasic tumoral formation with typically extensive leaf-like papillary structures growing toward slit-like spaces under the skin. In immunohistochemical examination, more than $50 \%$ of epithelial cells showed a positive reaction with ER, PR, and panCK and a focal positive reaction with GCDFP-15. Myoepithelial cells showed a positive reaction with SMA, CD10, and WT-1. In this report, we underline the clinicopathologic features of PT localized to an unusual site, and also discuss its etiology, differential diagnosis in the light of the current literature.
\end{abstract}

Keywords: Vulva, phyllodes tumor, skin, benign

Cite this article as: Kilitci A, Arız O. Primary Benign Phyllodes Tumor of The Vulva: Case Report and Review of Literature. Eur J Breast Health 2019; 15(3): 196-199.

\section{Introduction}

Breast lesions can also be found in extramammary areas such as the axilla, anus, prostate, seminal vesicle, and vulva. These lesions include diagnoses such as fibrocystic disease, fibroadenoma, intraductal papilloma, hidradenoma papilliferum, lactating adenoma, and adenocarcinoma. It is still uncertain that proliferative mammary gland lesions in the anogenital region originate from ectopic breast tissue or from local adnexal structures. Phyllodes tumor (PT) accounts for less than $1 \%$ of all breast tumors and can also occur in the ectopic areas such as the vulva and axilla $(1,2)$. PT of the vulva is very rarely seen and has been reported in only 17 cases in English literature. The majority of cases were benign except for one borderline case, one malignant case and two low-grade malignant cases.

\section{Case Presentation}

A 41-year-old female patient (Gravida 3, Para 3) was admitted to our polyclinic due to a painless, slow-growing mass under the skin of the vulva which she first noticed 6 months ago. The mass was located between the labium majus and minus at the 5-6 o'clock position in the vulva. It was $3 \times 4 \mathrm{~cm}$ in size and mobile. No redness or ulceration was observed. In the patient's history, there were no breastfeeding, breast disease, menstrual irregularity, malignancy, hormonal contraception, and pregnancy. Laboratory tests (hormone panel, gynecologi$\mathrm{cal}$ and breast cancer markers) were within normal limits. The lesion was excised and sent to the pathology laboratory for histopathological examination with a preliminary diagnosis of fibroma. Macroscopic examination revealed a round, well-circumscribed, dirty-white, elastic, grooved and nodular lesion $3 \mathrm{~cm}$ in diameter under the skin and a $2.4 \times 1.5 \mathrm{~cm}$ skin ellipse on its surface. No necrosis or hemorrhage was observed (Figure 1). Microscopic examination revealed biphasic tumoral formation with typically extensive leaf-like papillary structures growing toward slit-like spaces under the skin (Figure 2). The slit-like spaces consisted of a double layer of epithelial and myoepithelial cells (Figure 3). The stromal component consisted of monomorphic pale spindle cells and was hypocellular. Normal breast tissues were not detected in the sections prepared from the whole material. There were no stromal overgrowth, nuclear atypia, heterologous differentiation, or mitotic activity. In immunohistochemical examination, more than $50 \%$ of epithelial cells showed a positive reaction with ER, PR, panCK 


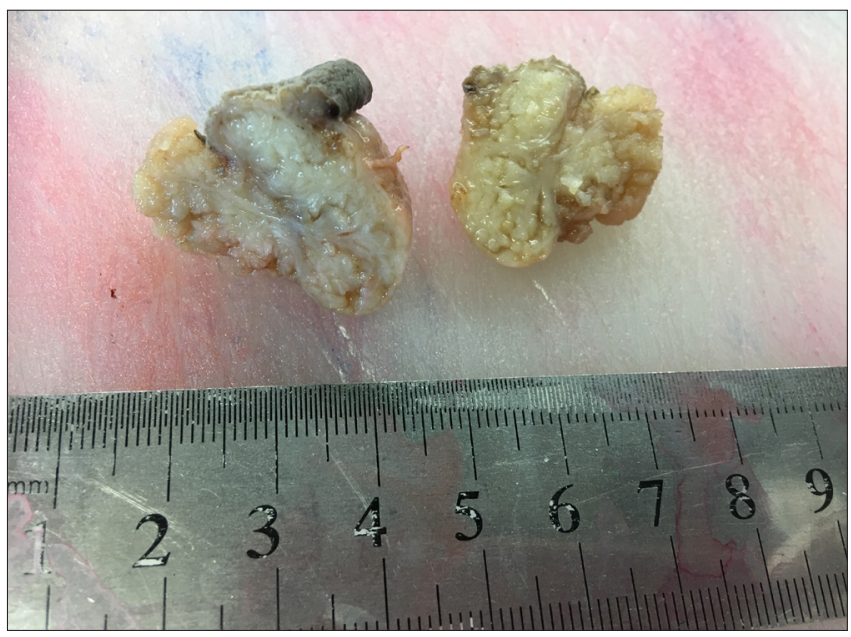

Figure 1. A round, well-circumscribed, dirty-white, elastic, grooved and nodular lesion $3 \mathrm{~cm}$ in diameter under the skin and a $2.4 \times 1.5 \mathrm{~cm}$ skin ellipse on its surface. No necrosis or hemorrhage was observed

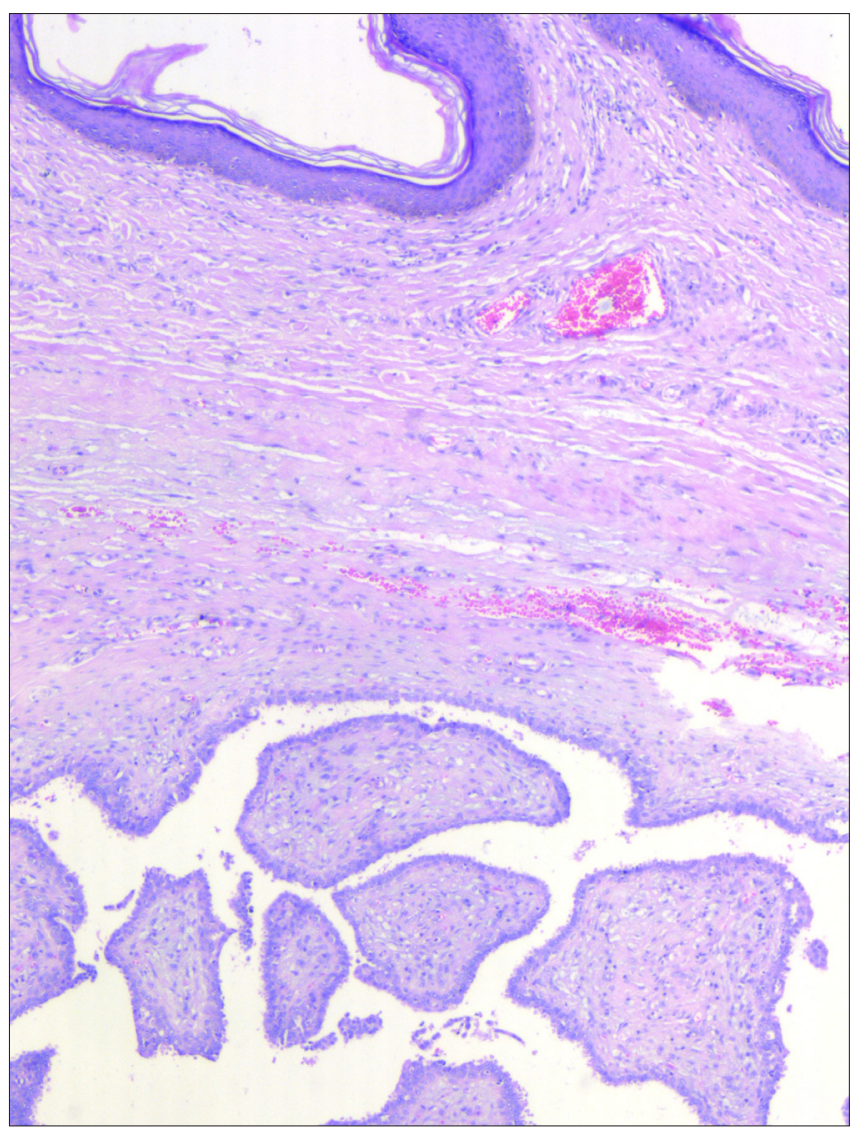

Figure 2. Typical extensive leaf-like papillary structures growing toward slit-like spaces under the skin $(H \& E, \times 50)$

and CK7 and a focal positive reaction with GCDFP-15 (Figure 4a, b). Myoepithelial cells showed a positive reaction with p63, SMA, CD10, and WT-1 (Figure 4c). CK20 and CDX2 were negative. The stromal component was negatively stained with these markers. In the presence of histomorphologic and immunohistochemical findings, the patient was diagnosed with benign PT. Because a portion of the lesion showed continuity in the surgical margin, the patient was followed for the risk of recurrence. Written informed consent was obtained from patient who participated in this study.

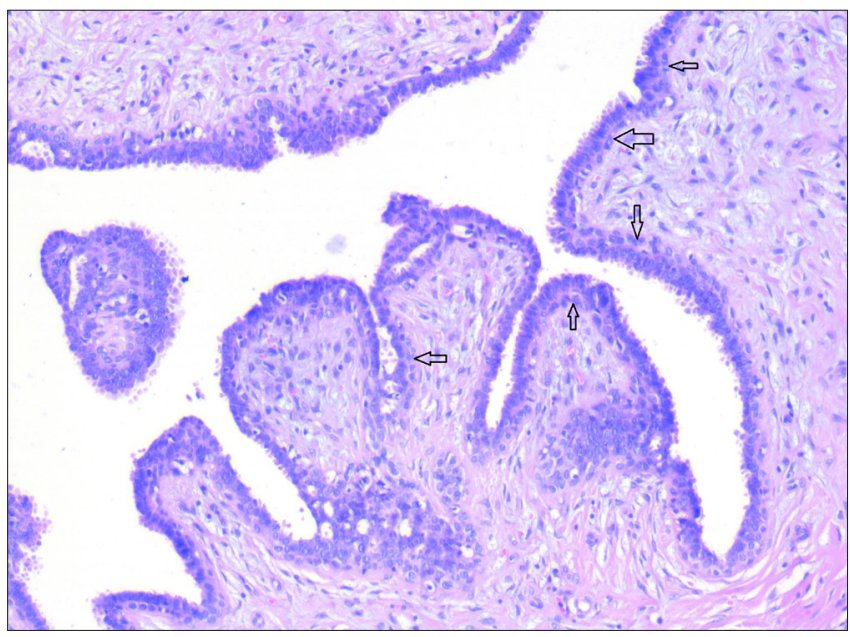

Figure 3. The slit-like spaces consisted of a double layer of epithelial and myoepithelial cells (myoepithelial cells were indicated by an arrow) (H\&E, x100)

\section{Discussion and Conclusion}

Two theories have been put forward to explain the cause of PT of the vulva. The first theory is the presence of ectopic breast tissue. The second theory is the presence of regional, specific and anogenital breastlike adnexal structures. The second theory is more accepted by the authors (3).

During the 4th and 6th week of fetal development, mammary buds develop as downgrowths from mammary crests, which are thickened strips of ectoderm extending from the axilla to the inguinal region. The breast tissue continues to develop in the normal pectoral region of the milk line, with regression of the remainder of the mammary ridges. The failure of this regression leads to the formation of ectopic breast tissue that can act as normal breast tissue or cause benign and malignant tumors. The actual incidence rate is unknown. Though this tissue is present at birth, it does not become evident until affected by female sex hormones at puberty, pregnancy, or lactation (4).

Van der Putte defined a specific variant of skin glands. In normal histology, the sebaceous glands are predominantly present on the medial surface of the labia majora, regardless of the presence of hair follicles. Van der Putte called these lesions mammary-like anogenital sweat glands, which consist of apocrine and eccrine glands and express estrogen and progesterone, rather than ectopic breast tissue. Similar to mammary glands, these glands have the capacity to branch into lobuli and to form acini. The origin of various neoplastic and reactive lesions of the anogenital region (including the vulva) which mimic mammary lesions has been explained with the presence of mammary-like glands. Moreover, it has been suggested that primordia of the mammary glands do not extend from the axillary-pectoral area to the vulva in human embryos, and that the number of ridges of mammary-like glands is greater than the number of residues of mammary-like glands, and that these glands are associated directly with cloacal derived tissues rather than breast lines. These have been reported to be localized in the interlabial sulcus, the paramedian area of the perineum and around the anus (5-7).

Mariappan et al. (8) have claimed that the presence of specific breast epithelium is not a prerequisite for the development of a tumor identical to mammary gland tumor, such as PT. PTs have also been 

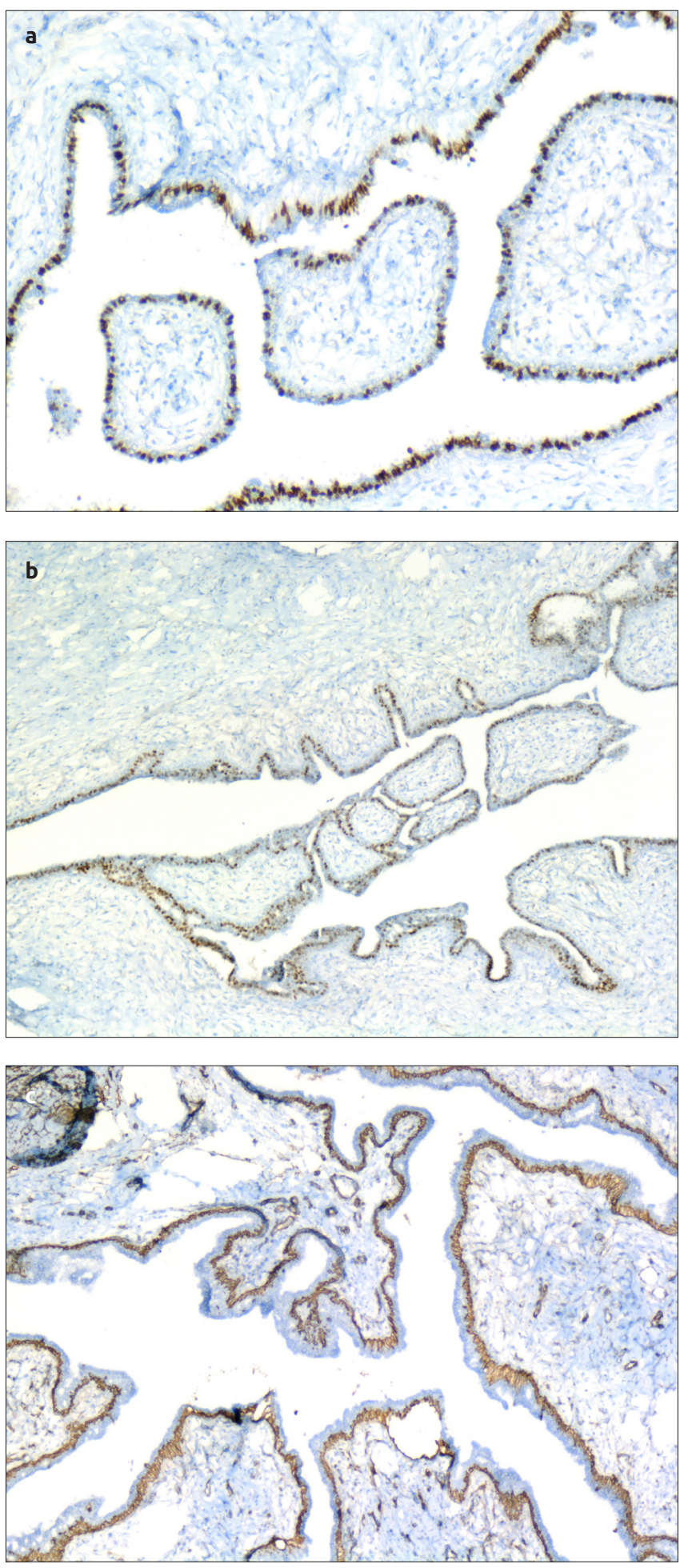

Figure 4. a-c. Immunohistochemical stains for PR, ER and SMA showed a positive reaction. A. PR x100, B. ER ×50, C. SMA x50

reported in caudal regions without milk lines, such as the perianal region, prostate, and seminal vesicle. Bostwick et al. (9) reported a series of 23 cases diagnosed with PT of the prostate. They showed that the cases did not give a positive reaction with estrogen and were stained with different immunohistochemical markers such as prostate specific antigen and prostatic acid phosphatase. They demonstrated that one case was positively stained with progesterone. They therefore hormonal factors might be effective in the pathogenesis of the tumor (9).

The presence of normal breast tissue or mammary-like glands around the lesion can give some clues about histogenesis (4). In our case, normal breast tissue or mammary-like glands were not observed within or around the lesion. However, the tumor was located between the labium majus and minus, and therefore it is likely to originate from the anogenital glands.

Patient with PT of the vulva are usually admitted with a one-sided, painless and solid mass. The labia majora, labia minora, and interlabial cleft are the most frequent sites of presentation. It involves both sides of the vulva in some cases admitted with itching, irritation, and dyspareunia (7). It is seen in the age range 17 to 69 years and commonly manifests in the $3 \mathrm{rd}$ and 4 th decades of life in women. The average age at the time of admission was 35.14 years. The largest tumor diameter varies from 0.7 to $6.6 \mathrm{~cm}$. The average tumor diameter is $3.47 \mathrm{~cm}$. The growth rate is variable. While some cases show rapid growth in the near future, other cases remain the same size for years and are incidentally detected. Macroscopically, it is dirty-white, homogeneous, solid and well-circumscribed, pushes surgical boundaries and has papillary projections or polypoid appearance $(2,3)$.

Phyllodes tumor of the vulva exhibits identical microscopic signs with its counterpart in the breast thanks to its biphasic pattern and leaflike configuration. Hyperplastic epithelium and pseudostratification can be observed. The stroma may show cellularity ranging from low to high. Significant nuclear pleomorphism is rare. There is no mitosis or lower. Immunohistochemically, secretory epithelial cells are positively stained with estrogen and progesterone, and myoepithelial cells are positively stained with p63, SMA and S100. The stroma is negatively stained with estrogen, progesterone, WT1, and CD10 and is positively stained with CD34, vimentin, and SMA. The Ki-67 proliferation rate can range from 1 to $15 \%$ (3).

Fibroadenoma is mainly considered in the differential diagnosis of PT of the vulva. The overlapping properties can be seen, and the separation of these entities from each other may be problematic. The characteristic leaf-like projections of PT are rarely described in fibroadenoma. Similar to PT of the breast, the tendency to become cystic, increased cellularity, cellular heterogeneity and stroma with cytological atypia may be more common in PT of the vulva (7). Papillary hidradenoma may show a complex papillary growth pattern such as PT but does not have a marked stromal component. Moreover, papillary structures are surrounded by double epithelial layer and have focal apocrine protrusions. Chondroid syringomas have cartilaginous islands within fibromyxoid stroma. In contrast to PTs, chondroid syringomas may have areas of squamous and sebaceous differentiation. Mullerian adenosarcoma of the cervix that secondarily involves the vulva may imitate a PT due to the formation of polypoid mass, a similar leaf-like architecture, and biphasic pattern. However, periglandular stromal condensation, increased stromal cellularity, and mitosis are more frequent (10).

Benign PT of the vulva is rarely seen in this localization and has homologous features with the breast histopathologically and immunohistochemically. However, its histogenesis has not yet been fully understood. In our case, we could not reveal histomorphologic findings that would definitely support one of the theories suggested in the pathogenesis. However, we think that the lesion can originate from anogenital mammary-like glands because it was located between the 
labium majus and minus and that a possible hormonal etiology may also play a role. Recurrence is rare with total resection of the tumor.

Informed Consent: Written informed consent was obtained from patient who participated in this study.

Peer-review: Externally peer-reviewed.

Author Contributions: Concept - A.K., O.A.; Design - O.A.; Supervision A.K.; Resources - A.K.; Materials - O.A., A.K.; Data Collection and/or Processing - O.A., A.K.; Analysis and/or Interpretation - A.K.; Literature Search - A.K.; Writing Manuscript - A.K.; Critical Review - A.K., OA.

Conflict of Interest: The authors have no conflicts of interest to declare.

Financial Disclosure: The authors declared that this study has received no financial support.

\section{References}

1. Moulla A, Hunt L, Shaikh H, Datta S. Phyllodes Tumor in the Vulva. Breast J 2017; 23: 476-478. (PMID: 28224691) [CrossRef]

2. Mannan AASR, Kahvic M, Aziz AHA. Phyllodes tumor of the vulva: report of a rare case and review of the literature. Am J Dermatopathol 2010; 32: 384-386. (PMID: 20514681) [CrossRef]
3. Lee S, Nodit L. Phyllodes tumor of vulva: a brief diagnostic review. Arch Pathol Lab Med 2014; 138: 1546-1550. (PMID: 25357118) [CrossRef]

4. Özbudak IH, Akkaya H, Akkaya B, Erdoğan G, Peştereli HE, Karaveli FŞ. Phyllodes Tumor of the Vulva: Report of Two Cases/Vulvanın Fillods Tümörü: İki Olgu Sunumu. Turk Patoloji Derg 2013; 29: 73-76. (PMID: 23354802) [CrossRef]

5. Van der Putte SC. Mammary-like glands of the vulva and their disorders. Int J Gynecol Pathol 1994; 13: 150-160. (PMID: 8005737) [CrossRef]

6. Wilkinson EJ, Hardt NS. Vulva. In: Mills SE. (Ed): Histology for pathologists. 3rd ed., Philadelphia, Lipppincott Williams and Wilkins 2007, 983-998.

7. Denlinger LN, Lokhandwala PM, Abendroth CS. Benign phyllodes tumor of the vulva: a case report and literature review. Rare tumors 2015; 7 : 148-150. (PMID: 26788277) [CrossRef]

8. Mariappan MR, Lagera JE, Fadare O, Sibley RK. A 69-year-old woman with a vulvar lesion. Phyllodes tumor of the vulva. Arch Pathol Lab Med 2006; 130: e11-e12. (PMID: 16390249)

9. Bostwick DG, Hossain D, Qian J, Neumann RM, Yang P, Young RH, Jones EC. Phyllodes tumor of the prostate: long-term followup study of 23 cases. J Urol 2004; 172: 894-899. (PMID: 15310992) [CrossRef]

10. Heffernan TP, Sarode VR, Hoffman B, Lea J. Recurrent phyllodes tumor of the vulva: a case report with review of diagnostic criteria and differential diagnosis. Int J Gynecol Pathol 2010; 29: 294-297. (PMID: 20407333) [CrossRef] 